\title{
Predicted Melt Curve and Liquid Shear Viscosity of RDX up to $30 \mathrm{GPa}$
}

\author{
Matthew P. Kroonblawd*[a] and H. Keo Springer ${ }^{[a]}$
}

\begin{abstract}
Recent grain scale simulations of HMX and TATB have shown that predictions for hot spot formation in high explosives are particularly sensitive to accurate determinations of the pressure-dependent melt curve and the shear viscosity of the liquid phase. These physics terms are poorly constrained beyond ambient pressure for the explosive RDX. We adopt an all-atom modeling approach using molecular dynamics (MD) simulations to predict the melt curve of RDX near to detonation conditions ( $30 \mathrm{GPa}$ ) and determine the shear viscosity of the liquid as a function of temperature and pressure above the melt curve. Phase-coexistence simulations were used to determine the melt curve, which is predicted to vary by almost $1100 \mathrm{~K}$ as the pressure increases from $0 \mathrm{GPa}$ to $30 \mathrm{GPa}$. Equilibrium MD simulations and the Green-Kubo formalism were used to obtain the pressure-temperature dependent shear viscosity. The shear viscosity of RDX is predicted to be of similar magnitude to the viscosity of TATB at low GPa-range pressures, and to be roughly an order of magnitude lower than the viscosity of HMX. The temperature dependence of the shear viscosity is Arrhenius at a given pressure, and the exponential pre-factor and activation term exhibit a strong, yet complicated, pressure dependence. An empirical pressure-temperature dependent function for RDX shear viscosity is developed that simultaneously captures a wide range of MD predictions while taking an analytic form that extrapolates smoothly beyond the fitted regime. The relative strength of the pressure and temperature dependencies of these two physics terms is found to be of similar magnitude for RDX, HMX, and TATB, which motivates incorporating these results in future RDX grain scale modeling.
\end{abstract}

\section{Keywords: Phase Diagrams, Rheology, Nitramines, Molecular Liquids, Multiscale Modeling}

\section{Introduction}

Initiation of high explosives is controlled through hot spots, which are localized regions of elevated temperature and energy that form when shock waves interact with microstructural defects such as pores and grain boundaries [1-4]. The physics of hot spot formation strongly depends on microstructure and elastic/inelastic material response, while hot spot evolution and growth into a self-sustained burn depends on a complex interplay between thermal conduction and reaction kinetics. Grain scale simulations with coarse grained particles [5-9] or continuum-based multiphysics models [10-14] are a primary tool for explicitly resolving the formation and growth of large hot spots thought to govern initiation response. Accuracy of grain scale model predictions depends on accurate determinations of a wide range of thermodynamic, mechanical, thermal, and chemical material properties that serve as direct inputs or calibrants. Parameters for these properties are often difficult to isolate in focused experiments at high pressures and temperatures so placeholder values are often used in grain scale models. All-atom modeling using molecular dynamics (MD) simulations has proven to be a robust tool for obtaining many of these missing material parameters [15-24]. Scale bridging between atomistic and grain scale simulations at commensurate

[a] M. P. Kroonblawd, H. K. Springer Energetic Materials Center

Lawrence Livermore National Laboratory,

700 East Avenue, Livermore, CA 94550 USA

${ }^{*}$ E-mail: kroonblawd1@IInl.gov

Supporting information for this article is appended at the end of the document. length and time scales has recently been used to calibrate and validate grain scale model terms through direct comparison [25-31]. Despite the promise of a one-and-done approach to calibration through scalebridging simulations, it is often more advantageous to gradually increase grain scale model complexity and independently determine physics terms due to the highly coupled and nonlinear nature of dynamic material response $[24,28,29]$. This is especially true for material parameters that vary significantly within the applicable temperature and pressure domain such as the melt curve and liquid shear viscosity [15, 21, 24]. We focus here on obtaining predictions for two key physics terms influencing hot spot formation, namely the melt curve and liquid shear viscosity, for the explosive RDX (hexahydro-1,3,5-trinitro-s-triazine) to near detonation conditions where there is a lack of data to parametrize and constrain grain scale models.

The melt curve plays a significant role in hot spot formation in high explosives on ultrafast time scales. Melting places an upper temperature limit on plastic work, which serves as a primary source for energy localization at hot spots and in shock heating of bulk crystal. In the case of HMX (octahydro-1,3,5,7tetranitro-1,3,5,7-tetrazocine), estimates of the melt curve based on the Lindemann law [32] were shown to be substantially lower than predictions obtained from MD simulations at GPa-range pressures [24]. Application of MD-derived melt curves for HMX [24] and TATB (1,3,5-triamino-2,4,6-trinitrobenzene) [21] in grain scale simulations of supported shocks were shown to effectively suppress melting during the collapse of micron- and sub-micron scale pores [24, 28, 29]. 
Predicted peak temperatures in these simulations were hundreds of Kelvin greater compared to analogous grain scale predictions with simpler melting models that give lower melting points at elevated pressures. Direct comparisons of grain scale simulations of shockinduced pore collapse to all-atom simulations showed that accurately capturing the melt curve was critical for consistency in predictions for the hot spot formation process [29]. While omitting melting physics altogether may work well in some situations, pressure unloading in unsupported shocks can lead to substantial melting of plastically worked crystal [24]. Complex variations in the pressure field in simulations of polycrystalline microstructures that might lead to melting are difficult to anticipate even for supported shocks. Accurate determinations of the melt curve are therefore necessary, regardless of whether melting is explicitly included or judiciously omitted in multiphysics explosive models.

Viscous flow can be a significant source for additional heating of melted material. Dynamic shear viscosity determines the transient deviatoric stresses that develop in a liquid in response to a shearing rate and the corresponding dissipative work. In some circumstances, the shear viscosity of a liquid will exhibit exponential dependence on temperature and pressure [16, 33-35] and it can also be a function of shearing rate. The pressure-temperature dependent shear viscosity of HMX and TATB at zero rate varies by orders of magnitude over a range of a few $\mathrm{GPa}$ and for a range of temperatures that are typical of shock initiation [15, $21,24]$. Simulations of viscous heating in melt pools formed at hot spots in HMX is predicted to push peak temperatures to values that are hundreds of Kelvin higher than in inviscid treatments [24]. Atomistic modeling predicts that plastic shear localization dynamically forms nanoscale shear bands of amorphous material in many explosives [9, 26, 36-40] and this response has been captured phenomenologically as melting in grain scale models $[10,13]$. Effective rate-dependent viscosities for $H M X$ in shear bands vary linearly in temperature [23] and approach zero-rate predictions $[15,24]$ for rates at or below $5 \times 10^{9} \mathrm{~s}^{-1}$. It remains an open question how best to model shear bands in explosives. Crossinspection of shear band temperatures in TATB $[40,41]$ against the predicted melt curve [21] indicates that the material in TATB shear bands falls well within the solid region of the phase diagram.

We focus here on characterizing the RDX solidliquid phase boundary and liquid phase shear viscosity, for which little is known under shock initiation and detonation conditions. RDX solid exhibits a rich polymorphism with six phases confirmed through diffraction experiments. The $\alpha$ form [42] and $\beta$ form [43] occur near normal conditions, although the $\beta$ form is highly metastable and readily converts to $\alpha$. Reversable transformations to high-pressure polymorphs have been identified near room temperature, including the $y$ form ( $P \sim 4 \mathrm{GPa}$ ) [44, 45], the $\delta$ form $(p \sim 18 \mathrm{GPa})$ [46], and the $\zeta$ form $(P \sim 28 \mathrm{GPa})$ [46]. A highly reactive $\varepsilon$ form has been isolated within a narrow range of elevated temperatures and pressures [47] that can be recovered at ambient pressure [48]. Most of these forms have verified orthorhombic space groups and exhibit discontinuous volume changes across a transition as well as differences in molecular conformations.

Considerably less is known about the melting point of RDX. The melting point is constrained by multiple experimental studies to a value of $477-478 \mathrm{~K}$ at ambient pressure [49-51]. Myint et al. [52] note one conference report by Nauflett et al. [53] that obtained somewhat larger values of the ambient-pressure melting point $480-484 \mathrm{~K}$; the equation of state model developed by Myint et al. yielded a melting point of $478.15 \mathrm{~K}$, in agreement with the other experiments. By comparison, there is only limited macroscopic evidence available for melting at elevated pressure. Dreger and Gupta [47] found a melting onset temperature of $488 \mathrm{~K}$ for $\alpha$-RDX at $0.65 \mathrm{GPa}$ that was quickly followed by reactions. Those authors identified spectroscopic and optical signatures for melting in diamond anvil cell experiments only up to $\sim 2 \mathrm{GPa}$, beyond which $\alpha-\mathrm{RDX}$ either decomposes directly or transforms to the reactive $\varepsilon$ form. It is important to note that the above measurements of solid-solid and solid-liquid transitions correspond to integrated macroscale observations on timescales of seconds or longer, which does not necessarily constrain the kinetics of transitions under dynamic loads on ultrafast time scales typical of shock initiation or detonation conditions.

Classical, non-reactive MD simulation provides what is perhaps the only practical route to determine the melt curve and liquid shear viscosity for non-meltcastable explosives such as RDX. While melting and viscous heating under pressure may play significant roles on ultrafast time scales, the melt is highly reactive which prevents direct experimental measurements. Similarly, while reactive MD simulations provide a solid basis for determining material response coupled to chemistry under these conditions [25-27], non-reactive simulations with a reasonable choice of force field (FF) allow for isolating a range of mechanical, thermal, and thermodynamic parameters of the reactant state. We use the well-established FF by Smith and Bharadwaj [54], which has found numerous applications to simulations of HMX and RDX under extreme conditions $[7,9,15,17-19,23,24,29,36,37,39,55-57]$. The predicted pressure-volume equation of state and Hugoniot are well described by this FF at GPa-range pressures, as is the relative ordering of the Gibbs energy as a function of pressure for the $\alpha$ and $y$ phases [55]. Predicted peak positions and line widths of $\alpha-R D X$ in the low frequency $(<10 \mathrm{THz})$ vibrational spectrum are close to experimental values [56], indicating that lattice modes are well described. Most of the predicted $\alpha-R D X$ elastic tensor coefficients near normal conditions are essentially the same as experiment within the scatter generated by the different MD methods used to compute these values [18]. The orientationally averaged thermal conductivity of $\alpha$-RDX predicted by the FF is close to pressed-powder experiments [17] and the predicted ordering of anisotropic conductivity along the three lattice directions matches single-crystals 
experiments [57]. Sellers et al. [19] found the SmithBharadwaj FF predicted the RDX melting point at atmospheric pressure to within $2.2 \%$ of experiment, lending confidence in the model for the present application.

We use MD simulations of phase coexistence $[58,59]$ to obtain the melt curve of RDX up to $30 \mathrm{GPa}$. Equilibrium MD simulations of the liquid phase and the Green-Kubo formalism [60] are then applied to predict the dynamic shear viscosity as a function of temperature and pressure above the melt curve. The remainder of the article is organized as follows. In section 2, we provide general simulation details and discussion on modifications to the Smith-Bharadwaj FF that improve simulation stability at high pressure conditions. Section 3 outlines the methods used to obtain the melt curve and discusses our predictions for RDX in the context of the limited experimental data and other computational determinations for the related explosives HMX and TATB. Section 4 outlines the methods used to predict the shear viscosity; a complicated temperature-pressure dependence is identified that we reduce to a straightforward analytic empirical form. Conclusions are summarized in Section 5.

\section{General Simulation Details}

Classical, non-reactive MD simulations were performed using the LAMMPS code [61] and a variant of the wellestablished force field (FF) for RDX and HMX by Smith and Bharadwaj [54]. This FF is of a class-I type and includes harmonic potentials for bonds, angles, and improper dihedrals and cosine series for proper dihedrals. Nonbonded interactions are modeled using the Buckingham potential (exp-6) with electrostatics between fixed partial charges on the nuclei. There are two differences between the original parameterization and that used here. These include a tuned harmonic potential for the $\mathrm{N}-\mathrm{O}$ bonds [62] and an additional $r^{-12}$ repulsive nonbonded interaction for each pair type that compensates for the divergence in the Buckingham potential at short separation [29, 63]. The additional repulsive potential was found to be necessary for simulations of the liquid phase at pressures above 10 GPa and leads to no appreciable difference in liquidphase density predictions or pairwise radial distribution functions at $10 \mathrm{GPa}$. Nonbonded terms were evaluated in real space up to an $11 \AA$ cutoff. Electrostatic terms were evaluated in real space using the Wolf potential [64] with an $11 \AA$ cutoff and a damping factor of $0.2 \AA^{-1}$. Additional implementation and validation details can be found in the Supporting Information.

Isochoric-isothermal (NVT) and isothermalisobaric (NPT) trajectories were integrated at various points in this study. Unless otherwise noted, isothermal simulations were performed using a Nosé-Hoover-style thermostat $[65,66]$ with a time constant of 100 fs. Isobaric simulations were performed using a NoséHoover-style barostat [67] with a time constant of 1000 fs and the optional drag parameter set to 1.0 (unitless). A triclinic barostat was used for simulations involving
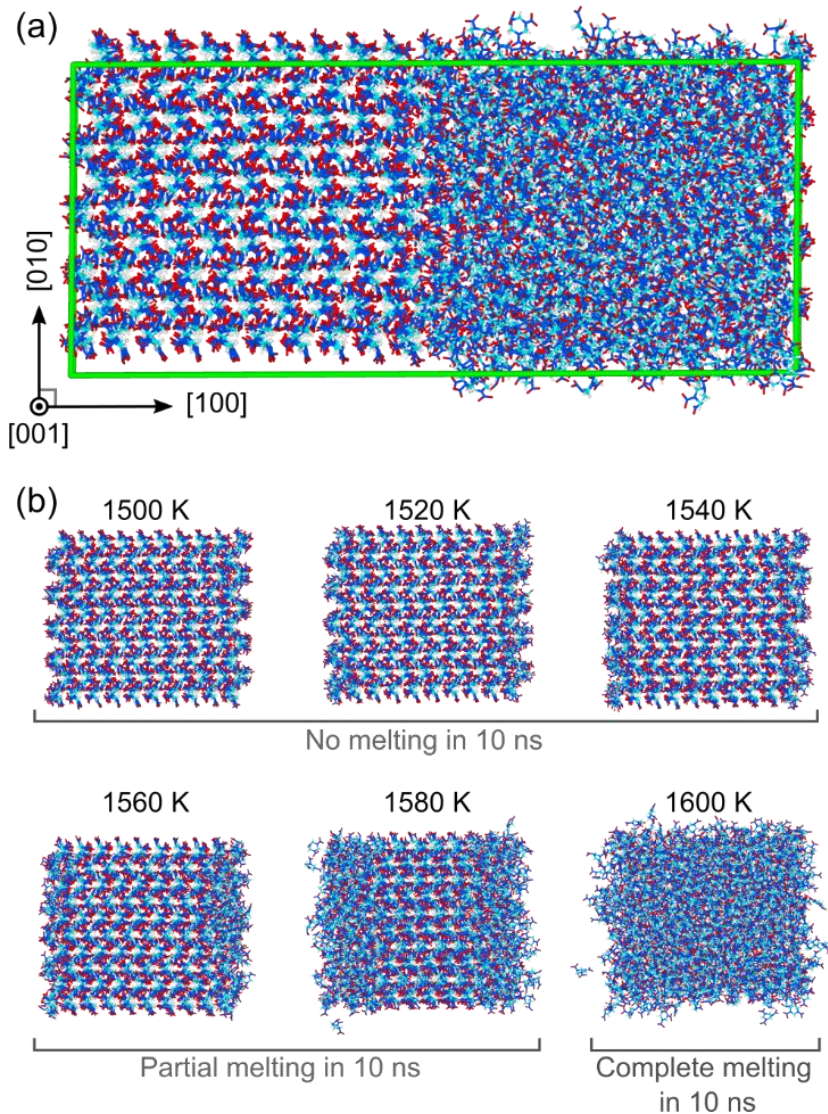

Figure 1. (a) Snapshot of the two-phase coexistence simulation cell in which a crystal slab is placed in contact with liquid. Atoms are colored cyan, blue, red, and white for $C, N, O$, and $H$ and the periodic boundaries are drawn with green lines. (b) Snapshots of the crystal region after 10 ns of NPT simulations at $30 \mathrm{GPa}$ and the indicated temperatures.

the crystal phase and an isotropic barostat was used for simulations of the pure liquid. All trajectories were integrated using a 0.5 fs time step. Simulation snapshots were rendered using OVITO [68].

\section{Melt Curve \\ 3.1 Methods}

The melt curve $T_{\mathrm{m}}(P)$ was determined as a function of pressure using MD simulations and the phasecoexistence approach $[58,59]$, which was previously applied to predict $T_{\mathrm{m}}(P)$ of $\mathrm{HMX}$ [24] and TATB $[69,21]$. In this approach, an initial metastable two-phase configuration is prepared in which a 2D-periodic crystal slab is placed in contact with an amorphous, liquid-like region in an overall 3D-periodic simulation cell. The specific cell used is shown in Figure 1(a) and includes a $5 \mathbf{a} \times 5 \mathbf{b} \times 5 \mathbf{c}$ a-RDX crystal supercell (1000 molecules) constructed using the structure of Choi and Prince [42] with an exposed (100) face in contact with 1000 liquid molecules. Procedures for constructing this cell closely follow earlier work on HMX [24]. The twophase simulation cell serves as the starting point for independent NPT simulations at dozens of $(T, P)$ states 
that bracket the melt curve. States above the melt curve lead to melting of the crystal region and typically involve an increase in the simulation cell volume. States below the melt curve generally show no loss of translational order for molecules in the crystal region, although typical MD simulations are usually not long enough to sample crystallization of the liquid region. All trajectories were $10 \mathrm{~ns}$ long, or less if the crystal region fully melted. We take $10 \mathrm{~ns}$ as an upper limit both due to the computational expense of these simulations and because $10 \mathrm{~ns}$ is a typical timescale for grain scale simulations of shock initiation $[10,13]$.

Changes in simulation cell volume $(V)$ with time $(t)$ are typically used as a metric for determining whether a simulation is above or below the melt curve, with $\dot{V}>0$ for $T>T_{\mathrm{m}}(P)$ and $\dot{V}<0$ for $T<T_{\mathrm{m}}(P)$. However, we found that this was not a reliable metric at higher pressures for RDX. For instance, all simulations at $30 \mathrm{GPa}$ exhibited $\dot{V}<0$ despite inspection of the trajectory showing clear melting of the crystal region. This likely owes to the initial two-phase simulation cell being prepared at a $0 \mathrm{GPa}$ pressure state. All melting point determinations were made based on visual inspection of the crystal region in the MD trajectories. Figure 1(b) shows snapshots of the crystal region at 30 $\mathrm{GPa}$ for different temperatures above and below the melting point. In this case, no melting was observed within 10 ns for $T \leq 1540 \mathrm{~K}$, some of the crystal layers melt for $1560 \mathrm{~K} \leq T \leq 1580 \mathrm{~K}$, and the crystal completely melts for $T \geq 1600 \mathrm{~K}$. Based on these results, we take the $30 \mathrm{GPa}$ melting point to be $1560 \mathrm{~K}$.

\subsection{Results}

Solid-liquid coexistence simulations were performed to determine the melting temperature of RDX at $1 \mathrm{~atm}$ (hereafter denoted $0 \mathrm{GPa}$ ), and elevated pressures of 1 , $3,5,10,20$, and $30 \mathrm{GPa}$. These simulations probe melting from the $\alpha$ phase even at high pressures and temperatures for which there are known solid-solid phase transitions. The Smith-Bharadwaj FF does not predict a prompt solid-solid $\alpha \rightarrow \gamma$ phase transition under hydrostatic loading up to at least $10 \mathrm{GPa}$ [55]. This contrasts with NPT-MD simulations performed using density functional theory (DFT) in which a homogeneous solid $\alpha \rightarrow \gamma$ transition occurs within 5 ps at $P=3.9 \mathrm{GPa}, T=300 \mathrm{~K}[70]$. This transition was observed for pressures as low as $2.75 \mathrm{GPa}$ in DFT simulations, which was attributed to the large stress fluctuations that arise in a small computational cell [70] and that may also influence the transition kinetics. Shock experiments show that the $\alpha \rightarrow \gamma$ transition requires an incubation time on the order of $100 \mathrm{~ns}$ [71] at $5.5 \mathrm{GPa}$. The timescale for decomposition chemistry arising from oriented shocks along three different directions is also on the order of 100s of nanoseconds for pressures between $7 \mathrm{GPa}$ and $20 \mathrm{GPa}[72,73]$. At the same time, kinetics for the $\alpha \rightarrow \varepsilon$ phase transition under hydrostatic conditions are on the order of hours and the transformation is followed promptly by chemical decomposition [47]. Thus, it is not unreasonable to expect the $\alpha$ phase to remain metastable and proceed
Table 1. Predicted RDX melting temperature at selected pressures and temperature bounds for phasecoexistence simulations.

\begin{tabular}{llll}
\hline$P(\mathrm{GPa})$ & $T_{\min }(\mathrm{K})$ & $T_{\max }(\mathrm{K})$ & $T_{\mathrm{m}}(\mathrm{K})$ \\
\hline 0 & 400 & 500 & 500 \\
1 & 500 & 640 & 580 \\
3 & 700 & 840 & 760 \\
5 & 800 & 940 & 900 \\
10 & 1000 & 1200 & 1140 \\
20 & 1300 & 1500 & 1420 \\
30 & 1500 & 1700 & 1560 \\
\hline
\end{tabular}

directly to the melt on ultrafast scales typical of shock initiation.

Independent NPT simulations were performed at different temperatures for each pressure value. Following a coarse assessment of the melt curve using short $\mathrm{O}(1 \mathrm{~ns})$ simulations at temperatures chosen in 100 $\mathrm{K}$ increments, the melting point was refined through 10 ns long trajectories performed in $20 \mathrm{~K}$ increments. Table 1 shows the minimum $\left(T_{\min }\right)$ and maximum $\left(T_{\max }\right)$ temperature values considered for these long $10 \mathrm{~ns}$ simulations as well as the predicted melting points $\left(T_{m}\right)$. As discussed above, the melting point was taken to be the temperature value for which at least one crystal layer lost translational order within $10 \mathrm{~ns}$ based on visual inspection of the trajectory (see Figure 1).

Focusing first on the melting point at $0 \mathrm{GPa}$, there is an anomalous transition to a pseudo-liquid state at some temperatures. Figure 2 shows snapshots of final crystal-region configurations at and below the predicted melting point of $500 \mathrm{~K}$. While there is a complete loss of rotational and translational order at 500 $\mathrm{K}$, the situation is more complicated at $460 \mathrm{~K}$ and $480 \mathrm{~K}$. A clear transformation takes place at $480 \mathrm{~K}$ to a state that exhibits rotational disorder but with translational order in two dimensions. Net displacement indicative of mass transport is also apparent, with several crystal molecules highlighted in the figure. A similar state is in the process of forming at $460 \mathrm{~K}$, with a distinct "reordered" region similar to the $480 \mathrm{~K}$ result seen in the lower right corner of the left-hand panel. No loss of the original crystal packing order is seen at $440 \mathrm{~K}$. This behavior was only found at $0 \mathrm{GPa}$, and even partially melted layers exhibited loss of both rotational and translational order at higher pressures.

The Smith-Bharadwaj FF yields a modest $4.6 \%$ overestimate of the melting temperature with the phasecoexistence approach at $0 \mathrm{GPa}$ relative to the experimental value of 477-478 K [49-51]. Dreger and Gupta [47] found that melting and decomposition of $\alpha$ $\mathrm{RDX}$ were closely linked at a pressure of $0.65 \mathrm{GPa}$ in diamond anvil cell experiments, with a melting onset temperature of $488 \mathrm{~K}$. Distinct changes in Raman spectra and optical images were observed to occur within minutes of melting that clearly indicated decomposition. Their melting onset value is somewhat lower than interpolations based on FF predictions $\left[T_{m}(0.65 \mathrm{GPa}) \approx 552 \mathrm{~K}\right]$, but it is also unclear whether melting and decomposition can be fully decoupled in the macroscopic observations. Decomposition of $\alpha-R D X$ 

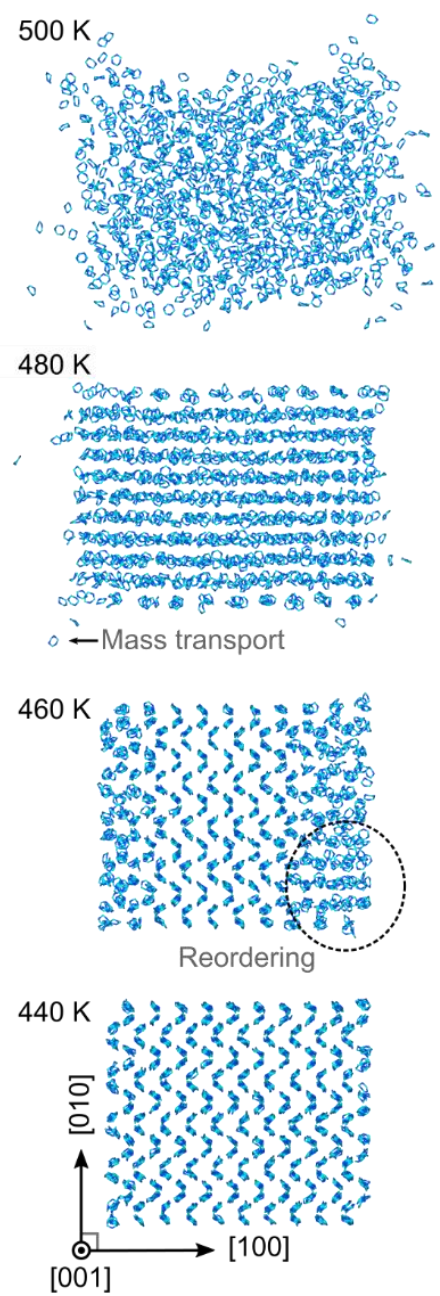

without any signs of initial melting was observed in those experiments above $2 \mathrm{GPa}$, as were transitions to the highly reactive $\varepsilon$ phase.

Several predictions for the melting temperature of $\alpha$ $\mathrm{RDX}$ at $0 \mathrm{GPa}$ have been reported in the literature that used the Smith-Bharadwaj FF. These include the determinations by Zheng and Thompson [74], who predicted melting from a superheated perfect crystal, and more recently be Sellers et al. [19] via more rigorous thermodynamic integration. Zheng and Thompson found that melting of the perfect crystal occurs at $510 \mathrm{~K}$ and is preceded by a subtle solid-solid phase transition near $490 \mathrm{~K}$. It is possible that their observed solid-solid transition bears some connection to the formation of a pseudo-liquid state in our simulations, but it was not possible to verify this based on the available information. The thermodynamic melting point determined by Sellers et al. was $488.75 \mathrm{~K}$, overestimating experiment by approximately $2 \%$. Thus, roughly half the error in the predicted melting point obtained via phase coexistence derives from fundamental errors in the FF description at $0 \mathrm{GPa}$, with the remainder arising from uncertainty due to coarseness in temperature sampling and the kinetics of melting.

The melting temperature is clearly a strong function of temperature and increases by nearly $1100 \mathrm{~K}$ as the pressure varies from 0 to $30 \mathrm{GPa}$. Similar to $\mathrm{HMX}$ and TATB, the predicted melt curve of RDX is well described by the empirical Simon-Glatzel equation [75],

$$
T_{m}(P)=T_{m, r e f}\left[1+\frac{P-P_{r e f}}{a}\right]^{1 / c} .
$$

Figure 2. Snapshots showing two views of the crystal region in phase-coexistence simulations of $R D X$ at 0 GPa near the melting point. Only the $C$ and $N$ atoms in the $R D X$ rings are rendered to enhance clarity of local rotational and translational order.

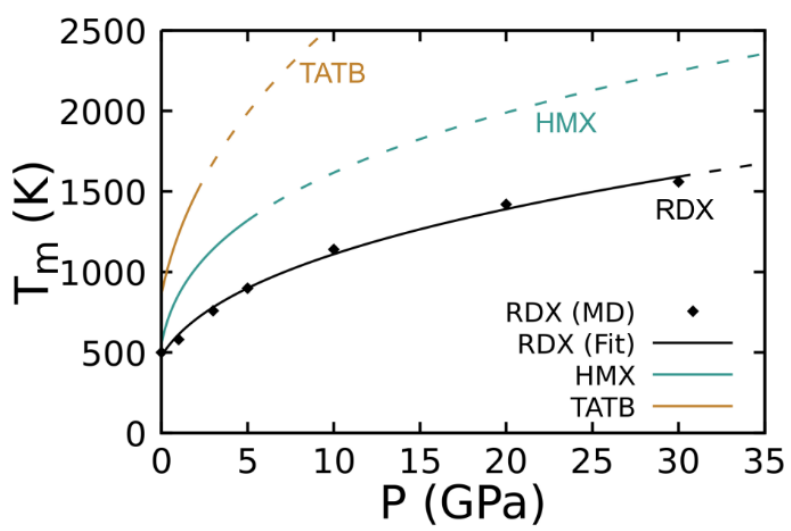

Figure 3. Predicted melt curves for RDX, HMX [24], and TATB [21]. Data points correspond to MD predictions determined using phase-coexistence simulations and lines to fits of the Simon-Glatzel equation to the MD data. Dashed lines indicate extrapolations beyond the highest pressure considered in MD simulations.

This form relates the melting point $T_{m, r e f}$ at a reference pressure $P_{r e f}$ with $a$ and $c$ as fitting parameters. Experimental values are taken as the reference point $\left(T_{m, r e f}=478 \mathrm{~K}, P_{r e f}=0.0001 \mathrm{GPa}\right)$ and the parameters were fit to minimize the root-mean-square error relative to the MD predictions for $P \geq 1 \mathrm{GPa}$. The best-fit parameters are $a=0.9631 \mathrm{GPa}$ and $c=2.8855$ (unitless).

Figure 3 shows a comparison of the MD predictions and Simon-Glatzel fit for the RDX melt curve to analogous MD-based Simon-Glatzel fits for the related explosives HMX [24] and TATB [21]. The melting temperature of RDX is substantially lower than either HMX or TATB and the differences generally increase with increasing pressure. The relative ordering of the predicted melting points for these explosives is consistent with the limited available experimental data, which gives $T_{m}^{\text {expt }}$ values of 478,551 , and $723 \mathrm{~K}$ for RDX [49-51], HMX [49], and TATB [76] at atmospheric pressure. Predictions for the HMX melt curve were obtained using the same FF used here for RDX, with the only exception being that the $\mathrm{C}-\mathrm{H}$ bond vibrations were held rigid in the MD simulations of HMX. Thus, differences between those two sets of predictions can be reasonably attributed to the increased molecular weight of HMX and the differing crystal packing structures. 


\section{Shear Viscosity \\ 4.1 Methods}

Dynamic shear viscosity of liquid RDX was determined as a function of temperature and pressure using NVT MD simulations and the Green-Kubo formalism [60]. The Green-Kubo formalism relates the autocorrelation of equilibrium shear stress fluctuations to the shear viscosity as

$$
\eta(T, P)=\frac{V(T, P)}{3 k_{B} T} \int_{0}^{\infty} \sum_{\alpha \beta=x y, x z, y z}\left\langle\sigma_{\alpha \beta}(t) \cdot \sigma_{\alpha \beta}(0)\right\rangle d t .
$$

Here, $\sigma_{\alpha \beta}(t)$ denotes the off-diagonal components of the stress tensor as a function of time, $\langle f\rangle$ denotes a time average of the stress autocorrelation function, $k_{B}$ is the Boltzmann constant, and $V(T, P)$ is the (constant) volume of the simulation cell that is determined by the equilibrium density at the specific $(T, P)$ state being simulated. Equilibrium $V(T, P)$ values were determined as the average over the last 250 ps of a 500 ps NPT trajectory. All viscosity calculation simulations were performed using cubic, 3D-periodic simulation cells containing 512 molecules.

We closely follow earlier work that determined the shear viscosity of HMX [24] and TATB [21], and so only outline practical considerations and extensions of the approach used to evaluate Equation 2. Three timescales arise including the period for sampling the stresses $\left(t_{\text {sample }}\right)$, the maximum time lag of the autocorrelation function $\left(t_{\text {block }}\right)$ that sets the upper limit of the integral, and the total simulation time $\left(t_{\text {sim }}\right)$. These must be respectively chosen to adequately sample stress fluctuations, be long enough for the autocorrelation to decay sufficiently close to zero and be long enough to obtain a good time average of the autocorrelation function. The fundamental sampling period was set to $t_{\text {sample }}=1 \mathrm{fs}$, but down sampling to 2 fs was found to yield essentially identical results and was used for the final analysis to improve the efficiency of computing the stress autocorrelation function. The last two timescales are coupled as the cumulative trajectory is split into $N$ contiguous time blocks of length $t_{\text {block }}=t_{\text {sim }} / N$.

Convergence of $\eta(T, P)$ is particularly sensitive to the upper limit of the integral, which we take to be $t_{\text {block }} / 2$. An example convergence study to determine an appropriate value of $t_{\text {block }}$ for the case $\mathrm{T}=1000 \mathrm{~K}$, $\mathrm{P}=0 \mathrm{GPa}$ is given in Figure 4. Panel (a) shows how the viscosity converges as a function of simulation time for selected values of $t_{\text {block}}$. Panel (b) shows average viscosity values as a function of block size computed over the second half of the trajectory, with the standard deviation taken as the uncertainty. Too short of $t_{\text {block }}$ yields a precise value that underestimates the true viscosity. In contrast, large $t_{\text {block }} \geq 40$ ps yield average values that are the same within uncertainty, but that uncertainty increases with block size as the number of samples $N$ decreases. We identify the best $t_{\text {block }}$ value for each state point as the value for which doubling the
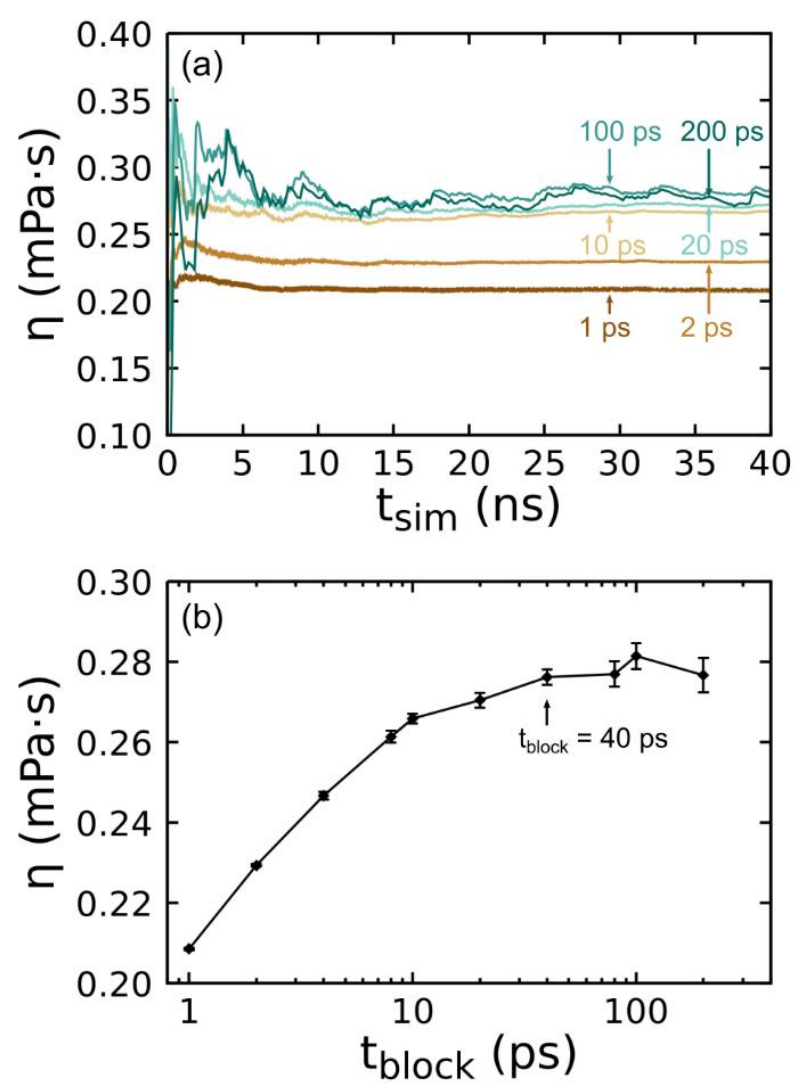

Figure 4. Convergence analysis for shear viscosity predictions at $P=0 \mathrm{GPa}$ and $T=1000 \mathrm{~K}$. (a) Time histories of the viscosity as a function of total simulation time $\left(t_{\text {sim }}\right)$ computed with selected block sizes $\left(t_{\text {block }}\right)$ indicated by labels and arrows. (b) Average viscosity value for $t \in[20 \mathrm{~ns}, 40 \mathrm{~ns}]$ plotted as a function of $t_{\text {block }}$.

block size yields the same average viscosity value within uncertainty.

Very long trajectories were needed for adequate sampling that approach hundreds of nanoseconds. We adopted an ensemble approach wherein many (typically 10-20) independent shorter trajectories were integrated for a single state point to obtain a large cumulative $t_{\text {sim }}$. Each trajectory was broken into contiguous blocks based on the maximum block size $t_{\text {block, max }}=4000 \mathrm{ps}$. An ensemble time history was assembled in which these 4000 ps blocks were intercalated in a round-robin fashion. All smaller $t_{\text {block }}$ values considered were a common divisor of $t_{\text {block,max }}$. Decorrelated starting points for each simulation in an ensemble were spawned from an NVT simulation performed using a (stochastic) Langevin thermostat $[77,78]$ with time constant 100 fs. Starting configurations were dumped every $100 \mathrm{ps}$. Note that the production NVT simulations used for evaluating $\eta(T, P)$ were performed using a Nosé-Hoover-style thermostat as stochastic thermostats can strongly alter dynamic properties such as transport coefficients [79]. 
Table 2. Fitted Andrade equation parameters for the temperature dependence of shear viscosity in liquid RDX, $H M X$, and TATB at selected pressures. Uncertainty in the last decimal place is given in parenthesis where available.

\begin{tabular}{|c|c|c|c|c|c|c|}
\hline \multirow[b]{2}{*}{$P(\mathrm{GPa})$} & \multicolumn{2}{|c|}{ RDX } & \multicolumn{2}{|c|}{ HMX } & \multicolumn{2}{|c|}{ TATB } \\
\hline & $\begin{array}{c}\eta_{0} \\
\left(\mathrm{mPa}^{*} \mathrm{~s}\right)\end{array}$ & $\begin{array}{c}E_{a} \\
(\mathrm{kcal} / \mathrm{mol})\end{array}$ & $\begin{array}{c}\eta_{0} \\
\left(\mathrm{mPa}^{*} \mathrm{~s}\right)\end{array}$ & $\begin{array}{c}E_{a} \\
(\mathrm{kcal} / \mathrm{mol})\end{array}$ & $\begin{array}{c}\eta_{0} \\
\left(\mathrm{mPa}^{*} \mathrm{~s}\right)\end{array}$ & $\begin{array}{c}E_{a} \\
(\mathrm{kcal} / \mathrm{mol})\end{array}$ \\
\hline 0 & $0.0038(2)$ & $8.61(7)$ & $8.4 \times 10^{-5}$ & 16.9 & $0.00120(1)$ & $11.74(2)$ \\
\hline 1 & $0.066(2)$ & $8.26(5)$ & $0.067(4)$ & $11.6(1)$ & $0.203(2)$ & $6.35(2)$ \\
\hline 3 & $0.123(4)$ & $10.91(8)$ & $0.17(2)$ & $13.7(3)$ & - & - \\
\hline 5 & $0.057(4)$ & $15.2(2)$ & 0.07 (2) & $19.3(8)$ & - & - \\
\hline 10 & $0.085(3)$ & $19.0(1)$ & - & - & - & - \\
\hline 20 & $0.20(2)$ & $21.9(3)$ & - & - & - & - \\
\hline 30 & $0.093(5)$ & $29.2(2)$ & - & - & - & - \\
\hline
\end{tabular}

\subsection{Results}

The shear viscosity of liquid RDX, $\eta(T, P)$, was obtained as a function of temperature and pressure using NVT simulations and the Green-Kubo relation at state points above the predicted melt curve for pressures of $0,1,3$, $5,10,20$, and $30 \mathrm{GPa}$. Equilibrium densities $\rho(T, P)$ used to prepare the NVT simulation cells and predicted $\eta(T, P)$ values are tabulated in the Supporting Information. The total simulation time $\left(t_{\text {sim }}\right)$ and block size $\left(t_{\text {block }}\right)$ needed to converge (or partially converge) the viscosity is also tabulated. We considered a maximum $t_{s i m, \max }=160 \mathrm{~ns}$ and a maximum $t_{\text {block, } \max }=4000 \mathrm{ps}$, which corresponds to a minimum of $N=40$ samples in the time average of the stress autocorrelation function. It was not possible to fully converge the viscosity for a small number of states near the melt curve, but partially converged viscosity values are included in plots below as they correspond to a reasonable lower bound. These partially converged states are specifically noted in the Supporting Information.

The shear viscosity is expected to depend exponentially on temperature for a given pressure, following an Arrhenius-like form known as the Andrade equation [80],

$$
\eta(T)=\eta_{0} \exp \left(\frac{E_{a}}{k_{B} T}\right)
$$

Weighted least-squares fits to Equation 3 were performed to obtain the pre-exponential factor and activation term and are listed in Table 2. While the form is Arrhenius, there is no conclusive physical interpretation of the activation term, nor is there a universal pressure-temperature dependent form for the viscosity of liquids [34]. Similar weighted fits to the Andrade equation were performed on literature values for HMX and TATB. Data for TATB was reported up to $2 \mathrm{GPa}$ and is thus only shown for comparison at the two lowest pressures [21]. The viscosity of $\mathrm{HMX}$ at $0 \mathrm{GPa}$ was determined by Bedrov et al. [15] and the values for 1, 3, and $5 \mathrm{GPa}$ were reported in Ref. [24]. Note that the
$0 \mathrm{GPa} H M X$ viscosity values did not have uncertainties, so unweighted fits were performed in that case. Both sets of HMX data were determined using the SmithBharadwaj FF, although there were several differences in the FF implementation and analysis that are discussed below.

Focusing first on the low-pressure response, Figure 5 shows comparisons of the present predictions for
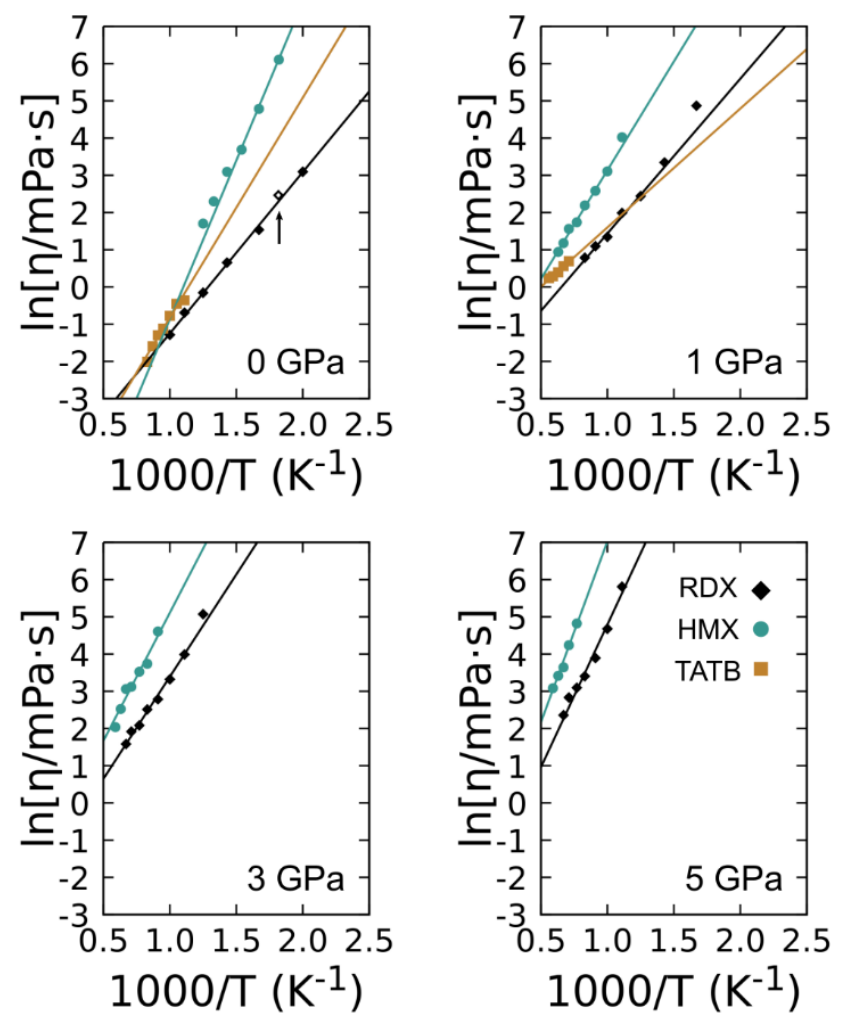

Figure 5. Comparison of predicted shear viscosities of RDX, HMX, and TATB at pressures up to $5 \mathrm{GPa}$. Data points correspond to $M D$ values and lines to weighted fits of the $M D$ results to Equation 3. The RDX viscosity value at $\mathrm{O} \mathrm{GPa}$ and $550 \mathrm{~K}$ determined by Izvekov and Rice [7] is shown as an open diamond symbol highlighted by an arrow. 
RDX to MD predictions for the related explosives HMX and TATB at pressures of $0,1,3$, and $5 \mathrm{GPa}$. Several trends are immediately apparent. The viscosities of HMX and RDX exhibit very similar slopes at 1,3 , and 5 $\mathrm{GPa}$, which is matched by their similar activation terms $\left(E_{a}\right)$. HMX viscosity is approximately one order of magnitude larger than RDX at any given temperature and pressure. In contrast, HMX and RDX have dissimilar slopes at $0 \mathrm{GPa}$ and the activation terms differ by a factor of two. The $0 \mathrm{GPa} H M X$ values were obtained using the Smith-Bharadwaj FF description in which all bonds were held rigid, unlike the higher pressure HMX values in which only $\mathrm{C}-\mathrm{H}$ bonds were held rigid. An empirical correction factor from the diagonal stress components [81] was also included in the $0 \mathrm{GPa}$ analysis to improve the rate of convergence that was not used for any of the other cases. Both of these differences were previously noted [24], but are more stark when contrasted against the present RDX data that were obtained with fully flexible molecules. Comparison of the RDX data across all four pressures and the HMX data above $0 \mathrm{GPa}$ show a general similarity in the activation term, indicating that the earlier $0 \mathrm{GPa}$ data for HMX is an outlier. The determination of RDX viscosity at $0 \mathrm{GPa}$ and $550 \mathrm{~K}$ by lzvekov and Rice [7] (open diamond symbol) is in very good agreement with the Andrade fit to our $0 \mathrm{GPa}$ predictions, which serves as an independent validation of our simulation and analysis procedure.

Comparison against TATB shows similar (if slightly larger) values for the viscosity of TATB compared to RDX, but the pressure dependence is clearly different. At $0 \mathrm{GPa}$, the magnitude of the activation term (slope) for TATB is larger than for RDX, but the opposite is true at $1 \mathrm{GPa}$. It is perhaps surprising RDX viscosity is closer to TATB than to HMX, which is more closely related to RDX both in terms of molecular shape and specific chemistry.

As alluded to above, there is no general form for the pressure or temperature dependence of liquid shear viscosity. Exponential forms can work well particularly within narrow temperature or pressure intervals $[34,35]$. These include the Andrade equation, which depends on the exponent of inverse temperature, and a pressure form that is exponential in pressure modulated by the Barus pressure-viscosity coefficient. Combined pressure-temperature exponential forms have been applied to explosive viscosity in the past $[33,16]$, but these forms were not able to reasonably capture the variation of RDX viscosity across the $30 \mathrm{GPa}$ pressure interval considered. Similarly poor descriptions were obtained with the empirical form used previously for HMX up to $5 \mathrm{GPa}$ [24]. (This form was an Andrade equation with power laws for a pressure-dependent prefactor and activation term.) More complex alternatives have been proposed [34,35], although these generally do not have a firm basis in theory.

Given the difficulty in finding a suitable functional form for the pressure-temperature dependent shear viscosity predictions, we took an empirical approach to arrive at a representation based on the Andrade equation that extrapolates smoothly beyond the states
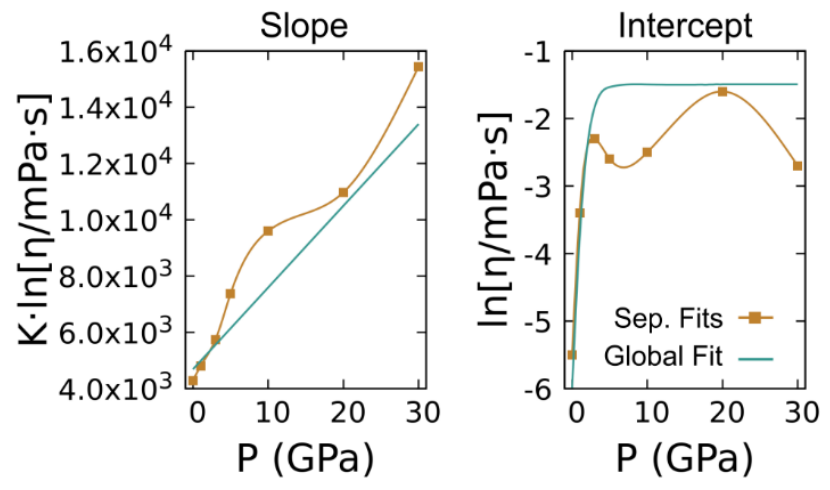

Figure 6. Pressure dependence of the slope and intercept terms for the Andrade equation fit to MD data for the RDX shear viscosity. Values obtained from separate unweighted fits of the MD data to Equation 3 (data points and spline curves) are compared to those obtained using a global fit with empirical pressuredependent forms for the slope and intercept (Eq. 4).

Table 3. Fitting parameters for a generalized pressuredependent Andrade equation describing the shear viscosity of RDX (see Equations 4-6).

\begin{tabular}{ll}
\hline Parameter & Fitted Value \\
\hline$A$ (K GPa- $\left.^{-1}\right)$ & 290.407 \\
$B(\mathrm{~K})$ & 4689.81 \\
$C$ (unitless) & 4.50278 \\
$D$ (GPa) & 1.83970 \\
$E$ (unitless) & -5.99842 \\
\hline
\end{tabular}

specifically inspected with MD simulations. Figure 6 shows the resulting pressure-dependent pre-factor (intercept) and activation term (slope) obtained from separate unweighted fits to the Andrade equation for each pressure series. These plots were used as a guide to identify appropriate pressure-dependent functional forms for the pre-factor and activation term in a generalization of Equation 3.

The slope parameter increases monotonically with pressure while the intercept exhibits several local maxima. This non-monotonic behavior is undesirable from the standpoint of obtaining a smoothly varying global surface for $\eta(T, P)$. Constraining the slope parameter to be linear in pressure suppressed the local minima in the intercept term leading to a function that increased rapidly with pressure before reaching a plateau (not shown). Based on this intermediate step, we chose the following form,

$\ln \left[\frac{\eta}{\mathrm{mPa} \cdot \mathrm{s}}\right]=(A \cdot P+B) \cdot \frac{1}{T}+\left[C \cdot \tanh \left(\frac{P}{D}\right)+E\right]$,

where the activation term is made linear in pressure,

$$
\frac{E_{a}}{k_{B}}=A \cdot P+B,
$$

and the pre-factor takes a hyperbolic tangent form,

$$
\ln \left(\frac{\eta_{0}}{\mathrm{mPa} \cdot \mathrm{s}}\right)=\left[C \cdot \tanh \left(\frac{P}{D}\right)+E\right] .
$$




\section{Conclusions}
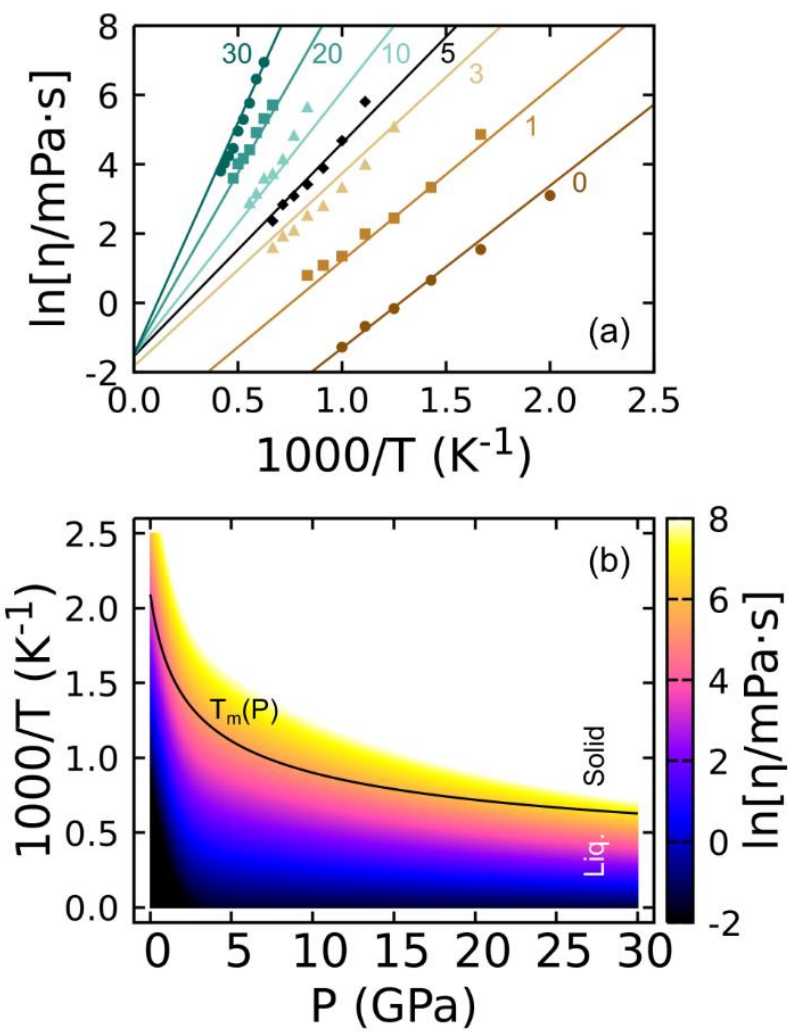

Figure 7. Optimized pressure-temperature-dependent shear viscosity $\eta(T, P)$ surface for liquid $R D X$ obtained using a modified form of the Andrade equation (Equation 4). (a) Comparison of $M D$ predictions (symbols) to the fitted form (lines) at each pressure. (b) Global surface for $\eta(T, P)$ with the predicted melt line $T_{m}(P)$ demarcating solid and liquid regions

The parameters $A, B, C, D$, and $E$ were obtained by a global least-squares fit to all the MD data and are collected in Table 3. Plots of the fitted pressuredependent slope (Equation 5) and intercept (Equation 6) are shown in Figure 5. Comparison against the data obtained from independent fits shows that both these forms reasonably capture the MD-predicted dependencies, including the rapid increase and plateau of the intercept with increasing pressure.

Figure 7 shows the optimized fit of the pressuredependent Andrade equation (Equation 4) for the $\eta(T, P)$ surface of RDX. Inspection of the line plots in panel (a) shows that the MD data for each pressure series is well-represented by the empirical form. Simultaneous changes in slope and intercept are accommodated and monotonic convergence of the intercept (pre-factor) to a constant value is clear. As expected from the functional form, the global surface in panel (b) shows smooth variation. Note that the function is not meaningful in the solid region of the diagram. The (undetermined) boundary between the liquid and vapor phase will also fall on this plot, although this boundary is likely of little consequence in reactive simulations as RDX would almost certainly react before vaporizing.
Accurate grain-scale models for simulating hot spot formation and growth depend on accurate determinations of basic thermodynamic, mechanical, thermal, and chemical material properties. Two of these terms, namely the pressure-dependent melt curve and liquid-phase shear viscosity, have been shown to exhibit a strong influence on predicted hot spot temperatures for HMX and TATB. This motivates an assessment of the RDX melting point, which is poorly constrained above ambient pressure, and the shear viscosity, which has not been characterized as a function of temperature or pressure. We use classical, non-reactive molecular dynamics (MD) simulations and an established force field (FF) model for RDX to predict the melt curve up to $30 \mathrm{GPa}$ and determine the temperature-pressure dependent shear viscosity.

Predictions for the melting point of RDX were obtained using the phase-coexistence approach. Results obtained here for the melting point at ambient pressure (1 atm) are within 5\% of experimental values and are similar to two other determinations made using the same FF but with different MD methods. The melt curve for RDX exhibits a significant pressure dependence, increasing by nearly $1100 \mathrm{~K}$ as the pressure increases from 0 to $30 \mathrm{GPa}$. The melting point of RDX is lower than the melting points of either $\mathrm{HMX}$ or TATB at a given pressure.

Dynamic shear viscosity was determined as a function of temperature at seven pressures ranging from 0 to $30 \mathrm{GPa}$ using equilibrium MD simulations and the Green-Kubo formalism. The temperature dependence at a given pressure exhibits Arrhenius-like behavior and is well-described by the Andrade equation. Comparison against similar results for liquid $\mathrm{HMX}$ and TATB at low $\mathrm{GPa}$-range pressures $(\leq 5 \mathrm{GPa})$ shows that the viscosity of RDX is similar in magnitude to TATB and roughly an order of magnitude lower than that of HMX. The exponential activation terms for RDX and HMX are similar at these pressures, with the difference in magnitude arising due to the pre-exponential factor. Both the activation term and pre-exponential factor exhibit a pressure dependence, which informs an empirical extension of the Andrade equation to obtain a global analytic surface for the viscosity as a function of temperature and pressure. This global function simultaneously captures a wide range of MD data points while taking a form that can be readily incorporated in grain scale models.

The relative strength of the pressuretemperature functional dependencies of the RDX melt curve and shear viscosity are similar in magnitude to recent determinations for HMX [24] and TATB [21]. Thus, it can be expected that grain scale simulation predictions of hot spot formation and growth in RDX will exhibit similar sensitivity as was found for those explosives [28, 29, 24]. Both the melt curve and shear viscosity were shown to independently alter peak hot spot temperatures by hundreds of Kelvin in HMX and TATB, which provides strong motivation for including the present results in future grain scale models of RDX. 


\section{Acknowledgements}

This work was performed under the auspices of the U.S. Department of Energy by Lawrence Livermore National Laboratory under Contract DE-AC52-07NA27344. It has been approved for unlimited release under document number LLNL-JRNL-829059-DRAFT.

\section{References}

[1] C. L. Mader, Initiation of detonation by the interaction of shocks with density discontinuities, Phys. Fluids 1965, 8, 1811-1816.

[2] J. E. Field, Hot spot ignition mechanisms for explosives, Acc. Chem. Res. 1992, 25, 489-496.

[3] C. M. Tarver, S. K. Chidester, A. L. Nichols, Critical conditions for impact- and shock-induced hot spots in solid explosives, J. Phys. Chem 1996, 100, 5794-5799.

[4] C. A. Handley, B. D. Lambourn, N. J. Whitworth, H. R. James, W. J. Belfield, Understanding the shock and detonation response of high explosives at the continuum and meso scales, Appl. Phys. Rev. 2018, 5, 011303.

[5] J. B. Maillet, L. Soulard, G. Stoltz, A reduced model for shock and detonation waves. II the reactive case, Europhys. Lett. 2007, 78, 68001.

[6] J. K. Brennan, M. LÍsal, J. D. Moore, S. Izvekov, I. V. Schweigert, J. P. Larentzos, Coarse-grain model simulations of nonequilibrium dynamics in heterogeneous materials, J. Phys. Chem. Lett. 2014, 5, 2144-2149.

[7] S. Izvekov, B. M. Rice, On the importance of shear dissipative forces in coarse-grained dynamics of molecular liquids, Phys. Chem. Chem. Phys. 2015, 17, 10795-10804.

[8] M. Lísal, J. P. Larentzos, M. S. Sellers, I. V. Schweigert, J. K. Brennan, Dissipative particle dynamics with reactions: application to RDX decomposition, J. Chem. Phys. 2019, 151, 114112.

[9] S. Izvekov, B. M. Rice, Bottom-up coarse-grain modeling of plasticity and nanoscale shear bands in $\alpha$ RDX, J. Chem. Phys. 2021, 155, 064503.

[10] R. A. Austin, N. R. Barton, J. E. Reaugh, L. E. Fried, Direct numerical simulation of shear localization and decomposition reactions in shock-loaded HMX crystal, J. Appl. Phys. 2015, 117, 185902.

[11] N. Grilli, C. A. Duarte, M. Koslowski, Dynamic fracture and hot-spot modeling in energetic composites, J. Appl. Phys. 2018, 123, 065101.
[12] O. Sen, N. K. Rai, A. S. Diggs, D. B. Hardin, H. S. Udaykumar, Multi-scale shock-to-detonation simulation of pressed energetic material: a meso-informed ignition and growth model, J. Appl. Phys. 2018, 124, 085110.

[13] H. K. Springer, S. Bastea, A. L. Nichols III, C. M. Tarver, J. E. Reaugh, Modeling the effects of shock pressure and pore morphology on hot spot mechanisms in HMX, Propel. Explos. Pyrotech. 2018, 43, 805-817.

[14] N. K. Rai, H. S. Udaykumar, Void collapse generated meso-scale energy localization in shocked energetic materials: non-dimensional parameters, regimes, and criticality of hotspots, Phys. Fluids 2019, 31, 016103.

[15] D. Bedrov, G. D. Smith, T. D. Sewell, Temperature-dependent shear viscosity coefficient of octahydro-1,3,5,7-tetranitro-1,3,5,7-tetrazocine (HMX): A molecular dynamics simulation study, J. Chem. Phys. 2000, 112, 7203-7208.

[16] R. Menikoff, T. D. Sewell, Constituent properties of HMX needed for mesoscale simulations, Combust. Theory Model. 2002, 6, 103-125.

[17] S. Izvekov, P. W. Chung, B. M. Rice, Nonequilibrium molecular dynamics simulation study of heat transport in hexahydro-1,3,5-trinitro-s-triazine (RDX), Int. J. Heat Mass Trans. 2011, 54, 5623-5632.

[18] D. E. Hooks, K. J. Ramos, C. A. Bolme, M. J. Cawkwell, Elasticity of crystalline molecular explosives, Propellants Explos. Pyrotech. 2015, 40, 333-350.

[19] M. S. Sellers, M. Lisal, J. K. Brennan, Free-energy calculations using classical molecular simulation: application to the determination of the melting point and chemical potential of a flexible RDX model, Phys. Chem. Chem. Phys. 2016, 18, 7841-7850.

[20] B. C. Barnes, K. W. Leiter, R. Becker, J. Knap, J. K. Brennan, LAMMPS integrated materials engine (lime) for efficient automation of particle-based simulations: application to equation of state generation, Model. Simul. Mater. Sci. Eng. 2017, 25, 055006.

[21] N. Mathew, M. P. Kroonblawd, T. Sewell, and D. L. Thompson, Predicted melt curve and liquid-state transport properties of TATB from molecular dynamics simulation, Mol. Sim. 2018, 44, 6113-622.

[22] G. Kumar, F. G. Van Gessel, D. C. Elton, P. Chung, Phonon lifetimes and thermal conductivity of the molecular crystal a-RDX, MRS Adv. 2019, 4, 2191-2199.

[23] M. Khan, C. R. Picu, Shear localization in molecular crystalcyclotetramethylene-tetranitramine (B-HMX): Constitutive behavior of the shear band, J. Appl. Phys. 2020, 128, 105902. 
[24] M. P. Kroonblawd, R. A. Austin, Sensitivity of pore collapse heating to the melting temperature and shear viscosity of HMX, Mech. Mat. 2021, 152, 103644.

[25] K. Lee, K. Joshi, S. Chaudhuri, D. S. Stewart, Mirrored continuum and molecular scale simulations of the ignition of high-pressure phases of $\mathrm{rdx}$, J. Chem. Phys. 2016, 144, 184111.

[26] M. A. Wood, D. E. Kittell, C. D. Yarrington, A. P. Thompson, Multiscale modeling of shock wave localization in porous energic material, Phys. Rev. B 2018, 97, 014109.

[27] M. N. Sakano, A. Hamed, E. M. Kober, N. Grilli, B. W. Hamilton, M. M. Islam, M. Koslowski, A. Strachan, J. Phys. Chem. A 2020, 124, 9141-9155.

[28] P. Zhao, S. Lee, T. Sewell, H. S. Udaykumar, Tandem molecular dynamics and continuum studies of shock-induced pore collapse in TATB, Propel. Explos. Pyrotech. 2020, 45, 1-28.

[29] P. Das, P. Zhao, D. Perera, T. Sewell, H. S. Udaykumar, Molecular dynamics-guided material model for the simulation of shock-induced pore collapse in $\beta$-octahydro-1,3,5,7-tetranitro-1,3,5,7-tetrazocine ( $\beta$ HMX), J. Appl. Phys. 2021, 130, 085901.

[30] C. A. Duarte, C. Li, B. W. Hamilton, A. Strachan, M. Koslowski, Continuum and molecular dynamics simulations of pore collapse in shocked $\beta$ tetramethylene tetranitramine $(\beta-\mathrm{HMX})$ single crystals, J. Appl. Phys. 2021, 129, 015904.

[31] M. P. Kroonblawd, B. W. Hamilton, A. Strachan, Fourier-like thermal relaxation of nanoscale explosive hot spots, J. Phys. Chem. C 2021, 125, 20570-20582.

[32] M. Ross, Generalized lindemann melting law, Phys. Rev. 1969, 184, 233-242.

[33] R. B. Frey, The initiation of explosive charges by rapid shear, $7^{\text {th }}$ Int. Det. Symp. 1981, 36-42.

[34] S. Bair, Temperature and pressure dependence of viscosity, in: Encyclopedia of Tribology (Eds.: Q. Jane Wang, Y.-W. Chung), Springer US, New York 2013, p. 3533.

[35] B. LotfizadehDehkordi, P.J. Shiller, G. L. Doll, Pressure- and temperature-dependent viscosity measurements of lubricants with polymeric viscosity modifiers, Front. Mech. Eng. 2019, 5, 18.

[36] E. Jaramillo, T. D. Sewell, A. Strachan, Atomic-level view of inelastic deformation in a shock loaded molecular crystal, Phys. Rev. B 2007, 76, 064112.

[37] M. J. Cawkwell, T. D. Sewell, L. Zheng, D. L. Thompson, Shock-induced shear bands in an energetic molecular crystal: Application of shock-front absorbing boundary conditions to molecular dynamics simulations, Phys. Rev. B 2008, 78, 014107.

[38] R. M. Eason, T. D. Sewell, Shock-induced inelastic deformation in oriented crystalline pentaerythritol tetranitrate, J. Phys. Chem. C 2012, 116, 2226-2239.

[39] R. M. Eason, T. D. Sewell, Molecular dynamics simulations of the collapse of a cylindrical pore in the energetic material $\alpha-R D X, J$. Dynamic Behavior Mater. 2015, 1, 423-438.

[40] M. P. Kroonblawd, L. E. Fried, High explosive ignition through chemically activated nanoscale shear bands, Phys. Rev. Lett. 2020, 124, 206002.

[41] M. P. Kroonblawd, B. A. Steele, M. D. Nelms, L. E. Fried, R. A. Austin, Anisotropic strength of single-crystal TATB, ChemRxiv 2021

[42] C. S. Choi, E. Prince, The crystal structure of cyclotrimethylene-trinitroamine, Acta. Cryst. 1972, B28, 2857-2862.

[43] D. I. A. Millar, I. D. H. Oswald, D. J. Francis, W. G. Marshall, C. R. Pulham, A. S. Cumming, The crystal structure of $\beta$-RDX-an elusive form of an explosive revealed, Chem. Commun. 2009, 562-564.

[44] N. Goto, H. Fujihisa, H. Yamawaki, K. Wakabayashi, Y. Nakayama, M. Yoshida, M. Koshi, Crystal structure of the high-pressure phase of hexahydro-1,3,5-trinitro1,3,5-triaxine ( $\mathrm{Y}-\mathrm{RDX})$, J. Phys. Chem. B 2006, 110, 23655-23659.

[45] A. J. Davidson, I. D. H. Oswald, D. J. Francis, A. R. Lennie, W. G. Marshall, D. I. A. Millar, C. R. Pulham, J. E. Warren, A. S. Cumming, Explosives under pressure - the crystal structure of $\mathrm{Y}$-RDX as determined by high-pressure X-ray and neutron diffraction, Cryst. Eng. Commun. 2008, 10, 162-165.

[46] C. Gao, X. Zhang, C. Zhang, Z. Sui, M. Hou, R. Dai, Z. Wang, X. Zheng, Z. Zhang, Effect of pressure gradient and new phases for 1,3,5-trinitrohexahydro-striazine (RDX) under high pressures, Phys. Chem. Chem. Phys. 2018, 20, 14374-14383.

[47] Z. A. Dreger, Y. M. Gupta, Phase diagram of hexahydro-1,3,5-trinitro-1,3,5-triazine crystals at high pressures and temperatures, J. Phys. Chem. A 2010, $114,8099-8105$.

[48] D. I. A. Millar, I. D. H. Oswald, C. Barry, D. J. Francis, W. G. Marshall, C. R. Pulham, A. S. Cumming, Pressure-cooking of explosives-the crystal structure of $\varepsilon$-RDX as determined by $\mathrm{X}$-ray and neutron diffraction, Chem Commun. 2010, 10, 5662-5664.

[49] P. G. Hall, Thermal decomposition and phase transitions in solid nitramines, Trans. Faraday Soc. 1971, 67, 556-562. 
[50] K. Kishore, Thermal decomposition studies on hexahydro-1,3,5-trinitro-s-triazine $(\mathrm{rdx})$ by differential scanning calorimetry, Propel. Explos. 1977, 2, 78-81.

[51] S. Zeman, Some predictions in the field of the physical thermal stability of nitramines, Thermochim. Acta 1997, 302, 11-16.

[52] P. C. Myint, M. A. McClelland, A. L. Nichols III, Application of the peng-robinson equation of state to energetic materials rdx and tnt: pure components, liquid mixtures, and solid mixtures, Ind. Eng. Chem. Res. 2016, 55, 2252-2266.

[53] G. W. Nauflett, D. Carlson, T. D. Austin, J. W. Brasch Sr., Effect of pressure and melting behavior on the combustion of propellants containing RDX and RDX admixtures. 16th JANNAF Combustion Meeting, Naval Postgraduate School, Monterey, CA, USA, 1979; pp 95111.

[54] G. D. Smith, R. K. Bharadwaj, Quantum chemistry based force field for simulations of HMX. J. Phys. Chem. 1999, B 103, 3570-3575.

[55] L. B. Munday, P. W. Chung, B. M. Rice, S. D. Solares, Simulations of high-pressure phases in rdx, J. Phys. Chem. B 2011, 115, 4378-4386.

[56] A. Pereverzev, T. D. Sewell, D. L. Thompson, Molecular dynamics study of the pressure-dependent terahertz infrared absorption spectrum of $\alpha$ - and $\gamma$-RDX, J. Chem. Phys. 2013, 139, 044108.

[57] R. Perriot, M. S. Powell, J. D. Lazarz, C. A. Bolme, S. D. McGrane, D. S. Moore, M. J. Cawkwell, K. J. Ramos, Pressure, temperature, and orientation dependent thermal conductivity of $\alpha-1,3,5$-trinitro-1,3,5triazinane ( $\alpha-R D X)$, arXiv 2021.

[58] J. R. Morris, C. Z. Wang, K. M. Ho, C. T. Chan, Melting line of aluminum from simulations of coexisting phases. Phys. Rev. B. 1994, 49, 3109-3115.

[59] Y. Zhang, E. J. Maginn, A comparison of methods for melting point calculation using molecular dynamics simulations. J. Chem. Phys. 2012, 136, 144116.

[61] S. Plimpton, Fast parallel algorithms for short-range molecular dynamics. J. Comput. Phys. 1995, 117, 1-19. LAMMPS is available at http://lammps.sandia.gov/.

[62] M. P. Kroonblawd, N. Mathew, S. Jiang, T. D. Sewell, A generalized crystal-cutting method for modeling arbitrarily oriented crystals in 3D periodic simulation cells with applications to crystal-crystal interfaces, Comput. Phys. Comm. 2016, 207, 232-242.

[63] D. Bedrov, O. Borodin, G. D. Smith, T. D. Sewell, D. M. Dattelbaum, L. L. Stevens, A molecular dynamics simulation study of crystalline 1,3,5-triamino-2,4,6- trinitrobenzene as a function of pressure and temperature, J. Chem. Phys. 2009, 131, 224703.

[64] D. Wolf, P. Keblinski, S. R. Phillpot, J. Eggebrecht, Exact method for the simulation of coulombic systems by spherically truncated, pairwise $\mathrm{r}^{-1}$ summation. J. Chem. Phys. 1999, 110, 8254-8282.

[65] S. Nosé, A unified formulation of the constant temperature molecular dynamics methods. J. Chem. Phys. 1984, 81, 511-519.

[66] W. G. Hoover, Canonical dynamics: equilibrium phase-space distributions, Phys. Rev. A. 1985, 31, 1695-1697.

[67] G. J. Martyna, D. L. Tobias, M. L. Klein, Constant pressure molecular dynamics algorithms, J. Chem. Phys. 1994, 101, 4177-4189.

[68] A. Stukowski, Visualization and analysis of atomistic simulation data with OVITO - the open visualization tool. Model. Simul. Mater. Sci. Eng. 2010, 18, 015012. OVITO is available at https://www.ovito.org.

[69] N. Mathew, T. D. Sewell, D. L. Thompson, Anisotropy in surface-initiated melting of the triclinic molecular crystal 1,3,5-triamino-2,4,6-trinitrobenzene: a molecular dynamics study, J. Chem. Phys. 2015, $143,94706$.

[70] D. C. Sorescu, B. M. Rice, RDX compression $\alpha \rightarrow$ $\gamma$ phase transition, and shock hugoniot calculations from density-functional-theory-based molecular dynamics simulations, J. Phys. Chem. C 2016, 120, 19547-19557.

[71] J. E. Patterson, Z. A. Dreger, Y. M. Gupta, Shock wave-induced phase transition in rdx single crystals, J. Phys. Chem. B 2007, 111, 10897-10904.

[72] J. E. Patterson, Z. A. Dreger, M. Miao, Y. M. Gupta, Shock wave induced decomposition of rdx: timeresolved spectroscopy, J. Phys. Chem. A 2008, 112, 7374-7378.

[73] N. C. Dang, Z. A. Dreger, Y. M. Gupta, D. E. Hooks, Time-resolved spectroscopic measurements of shockwave induced decomposition in cyclotrimethylene trinitramine ( $\mathrm{rdx}$ ) crystals: anisotropic response, J. Phys. Chem. A 2010, 114, 11560-11566.

[74] L. Zheng, D. L. Thompson, Molecular dynamics simulations of melting of perfect crystalline hexahydro1,3,5-trinitro-1,3,5-s-triazine, J. Chem. Phys. 2006, 125, 084505

[75] V. Kechin, Thermodynamically based meltingcurve equation. J. Phys.: Condens. Matter 1995, 7, 531535. 
Melt Curve and Viscosity of RDX

[76] A. Stolovy, E. C. Jones, J. B. Aviles, A. I. Namenson, W. A. Fraser, Exothermic reactions in TATB initiated by an electron beam, J. Chem. Phys. 1983, 78, 229-235.

[77] T. Schneider, E. Stoll, Molecular-dynamics study of a three-dimensional one-component model for distortive phase transitions, Phys. Rev. B 1978, 17, 1302-1322.

[78] B. Dünweg and W. Paul, Brownian dynamics simulations without gaussian random numbers, Int. J. Mod. Phys. C 1991, 2, 817-827.

[79] J. E. Basconi, M. R. Shirts, Effects of temperature control algorithms on transport properties and kinetics in molecular dynamics simulations, J. Chem. Theory Comput. 2013, 9, 2887-2899.

[80] E. C. DA C. Andrade, The viscosity of liquids, Nature 1930, 125, 309-310.

[81] P. J. Daivis, D. J. Evans, Transport coefficients of liquid butane near the boiling point by equilibrium molecular dynamics, J. Chem. Phys. 1995, 103, 42614265. 
Full Paper

M. P. Kroonblawd, H. K. Springer 


\section{Supporting Information}

\section{Predicted Melt Curve and Liquid Shear Viscosity of RDX up to $30 \mathrm{GPa}$}

Matthew P. Kroonblawd* and H. Keo Springer 


\section{Force Field Validation}

Non-Coulombic pairwise interactions in the Smith-Bharadwaj force field (FF) [1] were modeled using the Buckingham potential (exp-6), which diverges to minus infinity at very small atom-atom separation distances. This potential has the form

$$
U_{o l d}(r)=A \cdot \exp [-B r]-\frac{C}{r^{6}}
$$

Even very infrequent sampling of close contacts at high temperature-pressure conditions can result in simulation instability. We adopted an approach used by others $[2,3]$ in which an additional short-ranged repulsive potential was added to compensate for the divergence at short separation distance. This modified pairwise interaction potential was

$$
U_{n e w}(r)=A \cdot \exp [-B r]-\frac{C}{r^{6}}+D\left(\frac{12}{B r}\right)^{12}
$$

which includes an additional $\mathrm{r}^{-12}$ factor. The parameter $D$ was set to $5 \times 10^{-5} \mathrm{kcal} / \mathrm{mol}$ for all pair interaction types and was the same value used for a similar FF for TATB [2]. We verified that this modified FF yielded the same equilibrium density for the $(1500 \mathrm{~K}, 10 \mathrm{GPa})$ state point where both FF versions could be stably applied.

Figure S1 shows additional validation of the radial distribution functions (RDFs) for each pair interaction type at the $(1500 \mathrm{~K}, 10 \mathrm{GPa})$ state point that were obtained from the last $1 \mathrm{~ns}$ of a $2 \mathrm{~ns}$ NVT simulation. The two FF versions predict essentially identical RDFs for each pair type, indicating that the potential of mean force for each interaction type is unperturbed by the additional repulsive potential. The largest difference was seen for the first peak in the $\mathrm{N}$ (ring)-C interaction type, which may arise due to a very subtle distortion of the ring. 


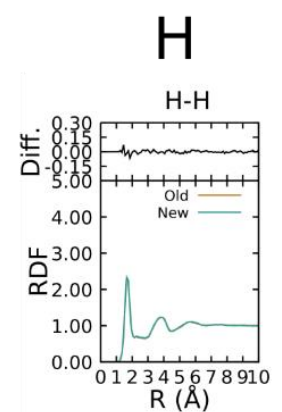

$\mathrm{N}\left(\mathrm{O}_{2}\right) \quad \mathrm{N}($ ring $)$
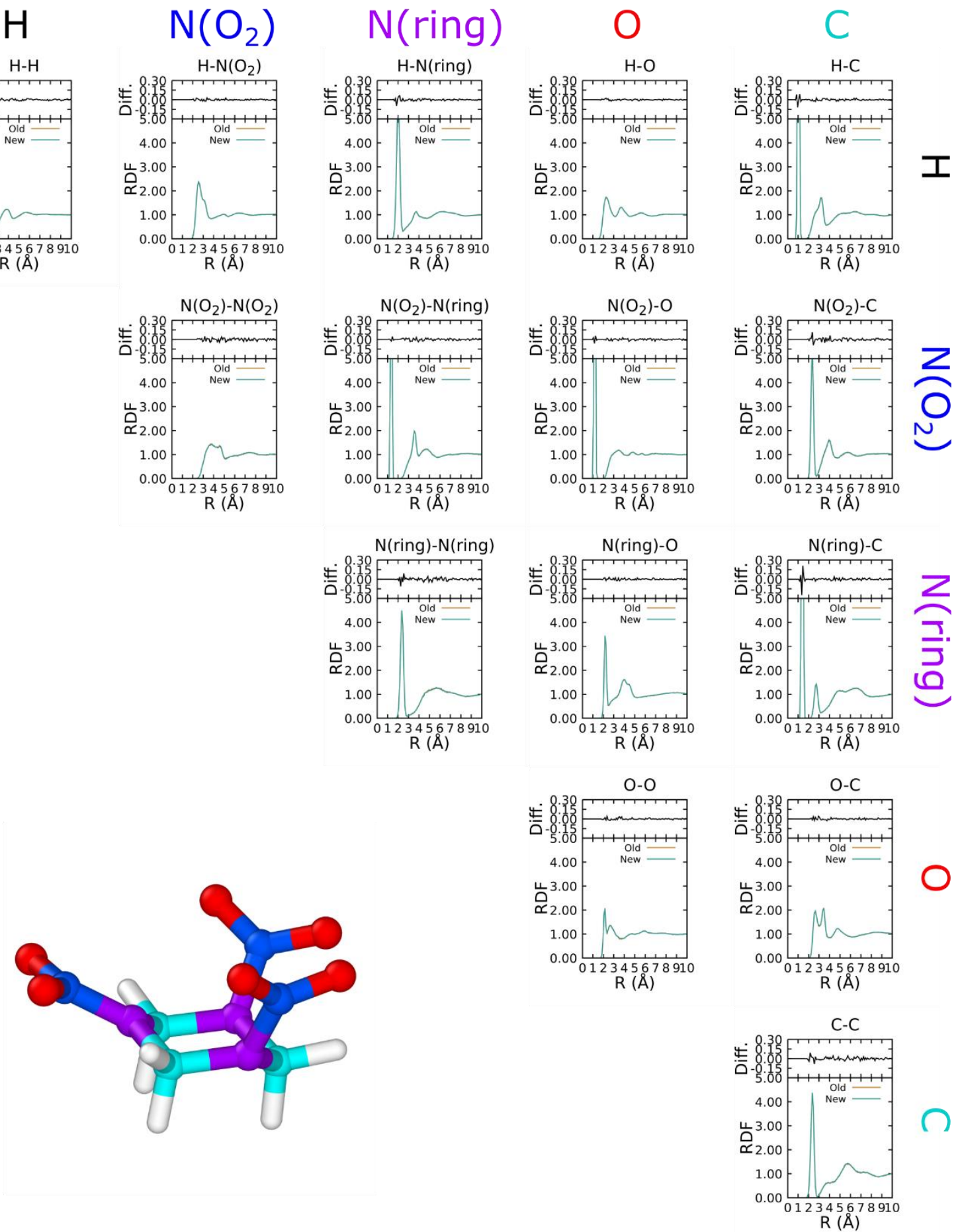

Figure S1: Comparison of radial distribution functions and differences for liquid RDX computed with the old FF parameterization and with the new repulsive $\mathrm{r}-12$ term, shown as a matrix of pair interaction types. 


\section{Tabulated Shear Viscosity Data}

\begin{tabular}{|c|c|c|c|c|c|c|c|}
\hline $\begin{array}{c}\mathrm{P} \\
(\mathrm{GPa})\end{array}$ & $\begin{array}{c}\mathrm{T} \\
(\mathrm{K})\end{array}$ & $\begin{array}{l}\text { Density } \\
\text { (g/cc) }\end{array}$ & $\begin{array}{c}\text { t_sim } \\
\text { (ns) }\end{array}$ & $\begin{array}{c}\text { t_block } \\
\text { (ps) }\end{array}$ & $\begin{array}{l}\text { Viscosity } \\
\text { (mPa*s) }\end{array}$ & $\begin{array}{l}\text { Uncertainty } \\
\left(\mathrm{mPa}^{*} \mathrm{~s}\right)\end{array}$ & $\begin{array}{c}\text { Fully } \\
\text { Converge? }\end{array}$ \\
\hline 0.0001 & 500 & 1.575 & 160 & 2000 & 22.011 & 0.835 & yes \\
\hline 0.0001 & 600 & 1.497 & 80 & 800 & 4.672 & 0.163 & yes \\
\hline 0.0001 & 700 & 1.412 & 50 & 400 & 1.914 & 0.043 & yes \\
\hline 0.0001 & 800 & 1.319 & 40 & 200 & 0.851 & 0.015 & yes \\
\hline 0.0001 & 900 & 1.211 & 40 & 200 & 0.504 & 0.012 & yes \\
\hline 0.0001 & 1000 & 1.063 & 40 & 40 & 0.277 & 0.004 & yes \\
\hline 1 & 600 & 1.771 & 160 & 4000 & 129.679 & 5.499 & no \\
\hline 1 & 700 & 1.733 & 60 & 800 & 28.219 & 1.281 & yes \\
\hline 1 & 800 & 1.698 & 40 & 400 & 11.458 & 0.098 & yes \\
\hline 1 & 900 & 1.666 & 40 & 400 & 7.295 & 0.175 & yes \\
\hline 1 & 1000 & 1.634 & 40 & 200 & 3.868 & 0.033 & yes \\
\hline 1 & 1100 & 1.607 & 40 & 100 & 2.966 & 0.028 & yes \\
\hline 1 & 1200 & 1.579 & 40 & 100 & 2.193 & 0.016 & yes \\
\hline 3 & 800 & 1.933 & 160 & 4000 & 158.401 & 5.091 & no \\
\hline 3 & 900 & 1.912 & 160 & 2000 & 53.906 & 1.187 & yes \\
\hline 3 & 1000 & 1.890 & 120 & 2000 & 27.781 & 0.553 & yes \\
\hline 3 & 1100 & 1.870 & 40 & 800 & 16.296 & 0.255 & yes \\
\hline 3 & 1200 & 1.851 & 40 & 800 & 12.359 & 0.545 & yes \\
\hline 3 & 1300 & 1.832 & 40 & 400 & 8.017 & 0.186 & yes \\
\hline 3 & 1400 & 1.816 & 40 & 400 & 6.801 & 0.129 & yes \\
\hline 3 & 1500 & 1.799 & 40 & 100 & 4.866 & 0.056 & yes \\
\hline 5 & 900 & 2.051 & 160 & 4000 & 333.775 & 9.086 & no \\
\hline 5 & 1000 & 2.034 & 160 & 4000 & 108.105 & 4.005 & no \\
\hline 5 & 1100 & 2.017 & 160 & 2000 & 48.932 & 1.341 & yes \\
\hline 5 & 1200 & 2.001 & 160 & 2000 & 30.274 & 0.876 & yes \\
\hline 5 & 1300 & 1.986 & 160 & 2000 & 21.919 & 0.663 & yes \\
\hline 5 & 1400 & 1.971 & 100 & 2000 & 16.964 & 0.755 & yes \\
\hline 5 & 1500 & 1.957 & 40 & 400 & 10.644 & 0.476 & yes \\
\hline 10 & 1200 & 2.235 & 160 & 4000 & 282.850 & 7.595 & no \\
\hline 10 & 1300 & 2.223 & 160 & 4000 & 123.145 & 3.316 & no \\
\hline 10 & 1400 & 2.211 & 80 & 800 & 62.475 & 2.045 & yes \\
\hline 10 & 1500 & 2.199 & 80 & 800 & 41.124 & 1.136 & yes \\
\hline 10 & 1600 & 2.189 & 80 & 800 & 36.069 & 0.797 & yes \\
\hline 10 & 1700 & 2.179 & 80 & 400 & 23.417 & 0.224 & yes \\
\hline 10 & 1800 & 2.168 & 80 & 400 & 17.721 & 0.247 & yes \\
\hline 20 & 1500 & 2.490 & 160 & 2000 & 301.662 & 6.612 & yes \\
\hline 20 & 1600 & 2.482 & 160 & 2000 & 203.344 & 5.326 & yes \\
\hline 20 & 1700 & 2.473 & 160 & 2000 & 137.177 & 5.559 & yes \\
\hline 20 & 1800 & 2.465 & 160 & 800 & 82.620 & 4.471 & yes \\
\hline 20 & 1900 & 2.457 & 80 & 800 & 64.548 & 2.016 & yes \\
\hline 20 & 2000 & 2.449 & 80 & 800 & 55.118 & 1.780 & yes \\
\hline 20 & 2100 & 2.442 & 80 & 400 & 36.547 & 0.810 & yes \\
\hline 30 & 1600 & 2.682 & 160 & 4000 & 1031.734 & 38.125 & no \\
\hline 30 & 1700 & 2.675 & 160 & 4000 & 641.666 & 19.168 & no \\
\hline 30 & 1800 & 2.667 & 160 & 2000 & 316.747 & 7.572 & yes \\
\hline 30 & 1900 & 2.660 & 160 & 2000 & 197.733 & 5.570 & yes \\
\hline 30 & 2000 & 2.654 & 160 & 1000 & 143.240 & 2.563 & yes \\
\hline 30 & 2100 & 2.647 & 80 & 400 & 85.912 & 1.769 & yes \\
\hline 30 & 2200 & 2.640 & 80 & 400 & 69.565 & 1.136 & yes \\
\hline 30 & 2300 & 2.634 & 80 & 400 & 56.341 & 0.871 & yes \\
\hline 30 & 2400 & 2.628 & 80 & 400 & 45.059 & 0.485 & yes \\
\hline
\end{tabular}




\section{Acknowledgements}

This work was performed under the auspices of the U.S. Department of Energy by Lawrence Livermore National Laboratory under Contract DE-AC52-07NA27344. It has been approved for unlimited release under document number LLNL-JRNL-829059-DRAFT.

\section{References}

[1] G. D. Smith, R. K. Bharadwaj, Quantum chemistry based force field for simulations of HMX. J. Phys. Chem. 1999, B 103, 3570-3575.

[2] D. Bedrov, O. Borodin, G. D. Smith, T. D. Sewell, D. M. Dattelbaum, L. L. Stevens, A molecular dynamics simulation study of crystalline 1,3,5-triamino-2,4,6-trinitrobenzene as a function of pressure and temperature, J. Chem. Phys. 2009, 131, 224703.

[3] P. Das, P. Zhao, D. Perera, T. Sewell, H. S. Udaykumar, Molecular dynamics-guided material model for the simulation of shock-induced pore collapse in $\beta$-octahydro-1,3,5,7-tetranitro-1,3,5,7-tetrazocine ( $\beta$ HMX), J. Appl. Phys. 2021, 130, 085901. 